\title{
ENHANCED MULTI-OBJECTIVE VOTING DATA ENVELOPMENT ANALYSIS MODELS WITH COMMON SET OF WEIGHTS
}

\author{
MOHAMMAD IZADIKHAH* AND ERDAL KARAPINAR
}

\begin{abstract}
The important issue of the aggregation preference is how to determine the weights associated with different ranking places and DEA models play an important role in this subject. DEA models use assignments of the same aggregate value (equal to unity) to evaluate multiple alternatives as efficient. Furthermore, overly diverse weights can appear, thus, the efficiency of different alternatives obtained by different sets of weights may be unable to be compared and ranked on the same basis. In order to solve two above problems, and rank all the alternatives on the same scale, in this paper, we propose a multiple objective programming (MOP) approach for generating a common set of weights in the DEA framework. Also, we develop a novel model to make a maximum discriminating among candidates' rankings. Additionally, we present two scenarios to provide suitable strategies for solving the proposed MOP model.
\end{abstract}

MSC(2010): 90B50; 90C05; 90C70.

Keywords: Multi-objective programming, Voting, Aggregation of preferences, Data envelopment analysis, Common set of weights, Ranking.

\section{Introduction}

Ranking a group or social from a set of individual preferences plays a bold and important role in decision-making context. In this class of methodologies, usually there are some decision-makers (DMs) in which each DM, selects a subset of the alternatives and places them in a ranked order. Assume that there are a set $A=A_{1}, A_{n},(n>3)$, of $\mathrm{n}$ alternatives (candidates) that have been compared and assessed by a specified group of experts as DMs (Izadikhah and Saen [1])). For the purpose of evaluating, each DM should give their own opinion by selecting a subset of $(k)$ alternatives (or the complete set A) and ranking them from most to least preferred. One of the primary related methods is weighting scoring rules (WSR). This method

Date: Received: September 27, 2020, Accepted: October 24, 2020.

${ }^{*}$ Corresponding author. 
operates by obtaining a score that depends on the rank position of the candidate in the individual's order of preferences. As a result, the alternatives are ranked by the sum of obtained scores. The obtained final score for the ith alternative is computed as $V_{i}=\sum_{j=1}^{k} w_{j} v_{i j}$. Where the weight of jth rank-position votes is denoted by $w_{j}$ and the number of the $j$ th rank positions votes that is obtained by ith candidate is denoted by $v_{i j}$. In WSR method, the candidate with more votes in the first place is recognized as the best and is considered as the origin of the family of preference-aggregation methodologies. In this method, the weight of the first place is calculated as the number of alternatives and the weight of the second place is one less than the first place and so on. Cook and Kress [2] showed some models like WSR that apply an imposed set of weights may fail to obtain a correct evaluation. Cook and Kress [2] applied a data envelopment analysis (DEA) based methodology of Charnes et al. [3] for measuring the preference scores without imposing any fixed weights from the outset (see [4, 5, 6]. Their proposed method obtained the most favorable weights for each alternative but their method very often leads to more than one alternative as efficient. To avoid this drawback and to find a deserved winner among the efficient alternative, Cook and Kress[2], presented a model to maximize the gap between consecutive weights of the scoring vector and as a result only one alternative is left efficient alternative. After that and in order to rank the candidates, Green et al. [7] developed the cross-efficiency based DEA evaluation methodology. Same as them, Noguchi et al.[8] was also applied the cross-efficiency evaluation to rank the alternatives (see $[9,10,11]$ ). They could give a strong ordering constraint condition on weights. Inspiring from the concept of super-efficiency proposed in Andersen and Petersen [12] Hashimoto[13] developed an AR/exclusion model to discriminate among efficient candidates. Obata and Ishii [14]investigated about the drawback of the above methods in the presence of an inefficient alternative and presented another method without to need of any information about inefficient candidates to discriminate efficient alternatives. Foroughi and Tamiz [15] extended their procedure to rank non-DEA-efficient candidates. Wang et al. [16] and Zerafat Angiz, et al. [17] developed DEA based models for solving the preference voting system. In conventional DEA models each decision making unit is allowed to measure its efficiency with the weights that are only most favorable for itself. The problem that may be risen is the efficiency scores of different DMUs obtained by different sets of weights may be unable to be compared and ranked on the same basis [18]. Another problem is that these methods cannot provide a fully discrimination, because of the flexibility in the selection of weights, always there are more than one efficient DMU. To overcome these problems, authors suggested finding a common set of weights for DMUs, and as a result, a number of methodologies have been proposed in the DEA context[19]. For instance, Ganley and Cubbin [20] derived the common weights by maximizing the sum of the efficiencies of 
DMUs. Kao and Hung [21] developed a hybrid method based on multiple objective nonlinear programming and compromise solution approach to generate a common set of weights (see $[22,23])$. In order to determine a common set of weights, Wang et al. [24] suggested ranking DMUs by imposing a minimum weight restriction. The current study has some contributions as follows. A new variant of Cook and Kress [2] with some interesting properties are provided. The proposed model is extended to the multi-objective programming model to deal with common set of weights. Then, a new model for estimating the maximum discriminating factor among candidates is developed. In order to solve the obtained MOLP model, two scenarios with two linear one-objective models are presented. The numerical results indicate that the proposed models provide complete ranking among candidates. The structure of this paper is organized as follows. In the next section the literature review is given. We present our proposed methods and their properties in section 3 . In section 4 , a numerical example shows the capabilities of the proposed models and finally conclusions is presented in section 5 .

\section{Literature Review}

Here we review some important works that are related to our proposed methodology.

2.1. Voting via DEA. One of the high usage concepts in decision-making problems is evaluating and ranking candidates based on some individual preferences. In order to evaluating the preference scores, firstly, Cook and Kress[2] developed a DEA based model by calculating the most favorable weights for each candidate. The bad thing about their model was the case of finding more than one efficient alternative. They tried to overcome the problem by increasing the distance between consecutive weights in a way that only one candidate is recognized as efficient. After that, Hashimoto [13] suggested a super-efficiency ([12]) method to increase discrimination among efficient alternatives. Recently, Ebrahimnejad et al. [25] applied a DEA method alongside a simulation method for assessing efficiency score of each candidate. Oukil and Amin [26] exploited power of individual appreciativeness in developing a procedure that integrates cross-evaluation, preference voting, and ordered weighted averaging. Izadikhah and Farzipoor Saen [1] developed a preference aggregation algorithm and used it to solve location planning problem.

2.2. Common set of weights. The concept of common set of weights (CSW) in data envelopment analysis firstly developed by Cook and Kress [2] and Roll et al. [27]. By developing CSW based on some DEA models and by removing the distance between upper and lower limits of weights, Cook and Kress [20, 28] proposed a new methodology for solving a voting system. Sinuany-Stern and Friedman [29] tried to use DEA model to find a suitable CSW for providing a complete ranking. There are two different 
aspects in CSW. The methods of the first aspect use a single objective function. In this aspect, Liu and Hsuan Peng [19], Sun et al. [30] and Saati and Nayebi [31] are some instances for this aspect. The second aspect is methods that use multiple objective programming. In this aspect, the methods of Jahanshahloo et al. [32], Cook and Zhu [33], Ruiz and Sirvent [34], Carrillo and Jorge [35] and Salahi et al. [36] can be mentioned.

2.3. Review of Voting System Models. In this subsection we are going to review Cook \& Kress [2] and Obata \& Ishii's [14] models in preferential voting system.

2.3.1. Cook and Kress's model. It is assumed that, there are $n$ alternatives (candidates) in which each alternative $i(i=1, \ldots, n)$ receives some number $v_{i 1}$ of first place votes, $v_{i 2}$ of second place votes $, \ldots, v_{i} k$ of $k$ th place votes. In the other hand, $v_{i j}$ denotes the number of $j$ th-place ranks that candidate i occupies $(i=1, \ldots, n, j=1, \ldots, k)$. Thus we have a ranked voting data which is obtained when voters select and rank more than one alternative. Here, consider each voter selects $k(>0)$ alternatives from a set of $n(k)$ alternatives and ranks them from top to kth place. The preference score $V_{i}$ of the alternative $\mathrm{i}$ is introduced as a weighted sum of votes with certain weights, $w_{j}$ i.e. $v_{i}=\sum_{j=1}^{k} w_{j} v_{i j},(i=1, \ldots, n)$. By means of data envelopment analysis methodology, Cook and Kress [2] developed a method for estimating preference scores without imposing any fixed weights from outset. The idea behind their method for evaluating each alternative is assigning the most favorable weights to that alternative. Their developed model for assessing the alternative $p$ is as model (2.3.1).

$$
\begin{aligned}
V_{p}^{*}= & \operatorname{Max} \sum_{j=1}^{k} w_{j} v_{p j} \\
\text { s.t } & \left\{\begin{array}{l}
\sum_{j=1}^{k} w_{j} v_{i j} \leq 1 ; \quad i=1, \ldots, n, \\
w_{j}+w_{j+1} \geq d(j, \varepsilon) ; \quad j=1, \ldots, k, \\
w_{k} \geq d(k, \varepsilon) ;
\end{array}\right.
\end{aligned}
$$

where $d(., \epsilon)$ called the "discrimination intensity function" which is monotonic increasing in and also the non-negative parameter is called the discriminating factor, and satisfies $\mathrm{d}(., 0)=0$. The optimal score $V_{p}^{*}$ is the preference score of the alternative $\mathrm{p}$. In this method each alternative is regarded as a DMU (Decision Making Unit) in DEA. Thus, here each DMU has k outputs (ranked votes) and has only one input of unity. The proposed model is equivalent to the well-known DEA-AR model (See Thompson et al. [37]) such that the constraints $w_{j}-w_{j+1} d(j, \epsilon)$ represent the assurance region (AR). These constraints are introduced to show that the vote of the higher place have a greater importance than that of the lower place. For the discrimination intensity function $\mathrm{d}(.$, ) Cook and Kress [2] introduced three 
special cases of $d(., \epsilon): d(., \epsilon)=\epsilon, d(., \epsilon)=\frac{\epsilon}{j}$ and $d(., \epsilon)=\frac{\epsilon}{j !}$. Each of them introduces different winner.

2.3.2. Obata and Ishii's Model. Obata and Ishii [17] believe that to obtain the maximum score of each alternative we should use weight vectors of the same size. For this purpose, they normalized the most favorable weight vectors for each candidate. Their proposed model for evaluate alternative $p$ is as Model (2.3.2).

$$
\begin{aligned}
& \frac{1}{\widehat{V}_{p}^{*}}=\operatorname{Min} \quad\|w\| \\
& \text { s.t }\left\{\begin{array}{l}
\sum_{j=1}^{k} w_{j} v_{p j}=1, \\
\sum_{j=1}^{k} w_{j} v_{i j} \leq 1, \quad i=1, \ldots, n, \quad i \neq p, \\
w_{j}-w_{j+1} \geq d(j, \varepsilon), \quad j=1, \ldots, k-1, \\
w_{k} \geq d(k, \varepsilon) ;
\end{array}\right.
\end{aligned}
$$

The normalized preference score $\widehat{V}_{p}^{*}$ is obtained as a reciprocal of the optimal value and $\|$.$\| is a certain norm.$

2.3.3. Izadikhah and Farzipoor Saen [1]. Izadikhah and Farzipoor Saen [1] used the [38, 39] method and the Cook and Kress [2] method to present a new approach to rank candidates based on strong complementary slackness condition, discriminant analysis, and discriminating factor as Model (2.3.3).

$$
\text { s.t. } \quad \begin{cases}\sum_{i=1}^{n} w_{i} v_{i j}+s_{j}=1, & j=1, \ldots, n, \\ -\sum_{i=1}^{n} w_{i} P_{i j}+q_{j}=-\varepsilon, & j=1, \ldots, n, \\ \sum_{j=1}^{n} \lambda_{j} v_{i j}-\sum_{j=1}^{n} h_{j} P_{i j}-k_{i}=v_{i p}, & i=1, \ldots, n, \\ \lambda_{j}+s_{j} \geq \varphi, & j=1, \ldots, n, \\ h_{j}+q_{j} \geq \varphi, & j=1, \ldots, n, \\ w_{i}+k_{i} \geq \varphi, & i=1, \ldots, n, \\ \sum_{i=1}^{n} w_{i} v_{i p}-\sum_{j=1}^{n} \lambda_{j}+\varepsilon \sum_{j=1}^{n} h_{j}=0, & \\ \lambda_{j}, s_{j}, h_{j}, q_{j}, w_{i}, k_{i}, \varphi \geq 0 . & \forall i, j .\end{cases}
$$

where $P=\left[\begin{array}{ccccc}1 & -1 & 0 & \ldots & 0 \\ 0 & 1 & -1 & \ldots & 0 \\ \vdots & \vdots & \vdots & & -1 \\ 0 & 0 & 0 & \ldots & 1\end{array}\right]$ and $v$ and $q$ are slack vectors. The model (2.3.3) corresponds to the evaluation of candidate $p$. 
2.3.4. Izadikhah and Saen[40]. Izadikhah and Farzipoor Saen [40] combined the presented methods of [25] and [1] and present the following Model (?) the evaluate the performance of candidate $\mathrm{p}$. :

$$
\begin{gathered}
v_{p}^{*}=\max \sum_{j=1}^{k} \sum_{t=1}^{T} y_{t j} v_{p j}^{t} \\
\text { s.t. }\left\{\begin{array}{l}
\sum_{j=1}^{k} \sum_{t=1}^{T} y_{t j} v_{i j}^{t} \leq 1, i=1, \ldots, n, \\
\sum_{j=1}^{k} y_{t j} P_{s j} \geq \varepsilon, s=1, \ldots, k ; t=1, \ldots, T, \\
\sum_{t=1}^{T} y_{t j} Q_{r t} \geq \varepsilon, j=1, \ldots, k ; r=1, \ldots, T .
\end{array}\right.
\end{gathered}
$$

where

$$
P=\left[\begin{array}{ccccc}
1 & -2 & 0 & \ldots & 0 \\
0 & 2 & -3 & \ldots & 0 \\
\vdots & \vdots & \vdots & & -(k-1) \\
0 & 0 & 0 & \ldots & 1
\end{array}\right](k \times k)
$$

and

$$
Q=\left[\begin{array}{ccccc}
1 & -2 & 0 & \ldots & 0 \\
0 & 2 & -3 & \ldots & 0 \\
\vdots & \vdots & \vdots & & -(T-1) \\
0 & 0 & 0 & \ldots & 1
\end{array}\right](T \times T)
$$

\section{Enhanced Voting Models Based on CSW}

Model (2.3.1) evaluates candidates by using different sets of weights, i.e. one set for each DMU and this make the assessment unfair. So, we build a new model that evaluate all DMUs against one common set of weights.

3.1. New Equivalence Model. For this purpose, we reformulate the Model (2.3.1) as following Model (3.1.1).

$$
\begin{aligned}
& \delta_{p}^{*}=\operatorname{Max} \gamma_{p} \\
& \text { s.t. }\left\{\begin{array}{l}
\sum_{j=1}^{k} w_{j} v_{p j} \geq \gamma_{p}, \\
\sum_{j=1}^{k} w_{j} v_{i j} \leq 1 ; \quad i=1, \ldots, n, \\
w_{j}-w_{j+1} \geq \varepsilon ; \quad j=1, \ldots, k-1, \\
w_{k} \geq \varepsilon .
\end{array}\right.
\end{aligned}
$$

Below theorem indicates a useful property of the Model (3.1.1).

Theorem 3.1. In optimality of Model (3.1.1) we have $\gamma_{p}^{*}=\sum_{j=1}^{k} w_{j}^{*} v_{p j}$.

Proof. By contrary, let's assume that in optimality we have $\gamma_{p}^{*}<\sum_{j=1}^{k} w_{j}^{*} v_{p j}$, Clearly, if we define $\tau_{p}=\frac{\sum_{j=1}^{k} w_{j}^{*} v_{p j}-\gamma_{p}^{*}}{2}$ then $\dot{\gamma_{p}}=\gamma_{p}^{*}+\tau_{p}>\gamma_{p}^{*}$ is a feasible 
solution that is better than the optimal solution. This contradiction proves the theorem.

The following theorem guarantees that both models (2.3.1) and (3.1.1) are equal and can be used interchangeably.

Theorem 3.2. Models (2.3.1) and (3.1.1) are equal.

Proof. A simple calculation shows that the feasible region of Model (3.1.1) is a subset of feasible region of Model (2.3.1). Let's assume that the vector $\left(w^{*},{ }_{p}^{*}\right)$ is a feasible solution of Model (3.1.1). Hence, the vector $w^{*}$ is a feasible solution for Model (2.3.1). Now, let's consider the vector $\tilde{w}$ be the optimal solution of Model (2.3.1). So, based on the above discussion, we must have $\gamma_{p}^{*}=\sum_{j=1}^{k} w_{j}^{*} v_{p j} \leq \sum_{j=1}^{k} \tilde{w}_{j} v_{p j}$. In contrary let's assume that $\sum_{j=1}^{k} w_{j}^{*} v_{p j}<\sum_{j=1}^{k} \tilde{w}_{j} v_{p j}$, Obviously, $\left(\tilde{w}, \tilde{y}_{p}\right)$ is a feasible solution for Model (3.1.1), where $\tilde{\gamma_{p}}=\stackrel{*}{\gamma_{p}}+\frac{\sum_{j=1}^{k} \tilde{w}_{j} v_{p j}-\sum_{j=1}^{k} w_{j}^{*} v_{p j}}{2}>\stackrel{*}{\gamma_{p}}$ This contradiction proves the theorem.

3.2. Proposed Models based on CSW. The above-mentioned models evaluate each candidate by a different set of weights. Thus, factor weights are allowed to vary freely in each run of the model. However, decision maker may not accept to allocate different weights to a factor. One possible way to overcome this difficulty lies in the specification of a CSW.

3.2.1. Justification of the CSW-based Model. Hence, we develop the following new multiple objective programming (MOP) model (3.2.1) for evaluating preference scores of all candidates by considering CSW.

$$
\text { s.t. } \quad\left\{\begin{array}{l}
\sum_{j=1}^{k} w_{j} v_{i j} \geq \gamma_{i} ; \quad i=1,2, \ldots, n, \\
\sum_{j=1}^{k} w_{j} v_{i j} \leq 1 ; \quad i=1,2, \ldots, n, \\
w_{j}-w_{j+1} \geq \epsilon ; \quad j=1,2, \ldots, k-1, \\
w_{k} \geq \epsilon ;
\end{array}\right.
$$

The MOP Model (3.2.1) evaluates all n candidates simultaneously. Before providing a suitable solution strategy, we note to the following interesting property.

Theorem 3.3. After solving Model (3.2.1) we have $\gamma_{p}^{*} \leq \delta_{p}^{*}, \quad \forall p$.

Proof. Regarding the fact that the feasible region of Model (3.2.1) is a subset of Model (2.3.4), the proof is obvious. 
3.2.2. Calculating a suitable value for discriminating factor (epsilon). To increase the discrimination power of the proposed model, we should set a suitable value for discriminating factor (epsilon). In order to find such a convenient value for $\epsilon$, [41, 42, 43], presented a mixed integer non-linear programming model. We remove the non-linearity problem and propose an enhanced version by proposing the following new mixed integer linear programming (MILP) model:

$$
\begin{aligned}
& \varepsilon^{*}=\operatorname{Max} \varepsilon \\
& \text { s.t. }\left\{\begin{array}{l}
\sum_{j=1}^{k} w_{j} v_{i j}+\xi_{i}=1 ; \quad i=1,2, \ldots, n, \\
w_{j}-w_{j+1} \geq \varepsilon ; \quad j=1,2, \ldots, k-1, \\
w_{k} \geq \varepsilon \\
\sum_{i=1}^{n} \eta_{i}=n-1, \\
0 \leq \xi_{i} \leq \eta_{i} ; \quad i=1,2, \ldots, n, \\
\xi_{i} \in\{0,1\} ; \quad i=1,2, \ldots, n .
\end{array}\right.
\end{aligned}
$$

The proposed model guarantees that in optimality we have $0 \leq \xi_{i}^{*} \leq 1,(\forall i)$. This model calculates the optimal value of discriminating factor (epsilon) in a way that the most discrimination power is achieved.

Theorem 3.4. Model (3.2.2) is always bounded, i.e. $\varepsilon^{*}<\infty$.

Proof. From the first group of constraints of model (3.2.2), we have

$$
\sum_{j=1}^{k} w_{j} v_{i j} \leq 1, \quad(\forall i) \Rightarrow \exists M ; \forall j w_{j}<M .
$$

From the last two groups of constraints, we have $\varepsilon^{*} \leq M<\infty$

If we use the optimal value of the discriminating factor (epsilon) in model (3.2.2), the following MOP model (8) is obtained:

$$
\text { s.t. } \quad\left\{\begin{array}{l}
\sum_{j=1}^{k} w_{j} v_{i j} \geq \gamma_{i} ; \quad i=1,2, \ldots, n, \\
\sum_{j=1}^{k} w_{j} v_{i j} \leq 1 ; \quad i=1,2, \ldots, n, \\
w_{j}-w_{j+1} \geq \varepsilon ; \quad j=1,2, \ldots, k-1, \\
w_{k} \geq \varepsilon^{*} ;
\end{array}\right.
$$

Where $\varepsilon^{*}$ is optimal value of model (3.2.2). This model has the most discrimination power among candidates and can present a complete ranking.

3.2.3. Solving Strategy. The proposed Model (8) is a multi-objective programming model and there are various methods for converting it into the 
one-objective model. To solve the MOP Model (8), we consider two scenarios that lead to two new models. To this end, we use compromise approach and max-min methods.

\section{Scenario 1: Compromise Approach}

From the first two constraints of Model (8), we know the best value for each $\gamma_{i}$ is one. So, we can use the compromise solution approach to convert it to one-objective model as follows:

$$
\min \left(\sum_{i=1}^{n}\left(1-\gamma_{i}\right)^{p}\right)^{\frac{1}{p}}
$$

In which for $p=1$, we reach into the following linear programming model (3.2.3).

$$
\begin{aligned}
& \operatorname{Min}\left(\sum_{i=1}^{n}\left(1-\gamma_{i}\right)\right) \\
& \text { s.t. }\left\{\begin{array}{l}
\sum_{j=1}^{k} w_{j} v_{i j} \geq \gamma_{i} ; \quad i=1,2, \ldots, n, \\
\sum_{j=1}^{k} w_{j} v_{i j} \leq 1 ; \quad i=1,2, \ldots, n, \\
w_{j}-w_{j+1} \geq \varepsilon^{*} ; \quad j=1,2, \ldots, k-1, \\
w_{k} \geq \varepsilon^{*} ;
\end{array}\right.
\end{aligned}
$$

Model (3.2.3) has only one objective function and can easily be solved. By solving Model (3.2.3), the final score for each alternative can be obtained.

\section{Scenario 2: Max-Min Approach}

In the second scenario to solve MOP Model (8), we consider the max-min method to maximize the minimum performances. Using this idea, model (8) is converted into the following one-objective model.

$$
\begin{aligned}
& \operatorname{Max} a \\
& \text { s.t. }\left\{\begin{array}{l}
\gamma_{i} \geq a ; \quad i=1,2, \ldots, n, \\
\sum_{j=1}^{k} w_{j} v_{i j} \geq \gamma_{i} ; \quad i=1,2, \ldots, n, \\
\sum_{j=1}^{k} w_{j} v_{i j} \leq 1 ; \quad i=1,2, \ldots, n, \\
w_{j}-w_{j+1} \geq \varepsilon^{*} ; \quad j=1,2, \ldots, k-1, \\
w_{k} \geq \varepsilon^{*} ;
\end{array}\right.
\end{aligned}
$$

Similarly, model (3.2.3) is also a programming model with only one objective function and can easily be solved. By running model (3.2.3), the final score for each alternative can be obtained.

\section{Numerical comparison}

Here, we analyze our proposed models through a numerical example. For this purpose, we take dataset of [14] which involves seven candidates. 150 
voters evaluated these candidates and determined the first and second rank. Votes are summarized in Table 1.

\begin{tabular}{|c|c|c|}
\hline Candidates & First rank & Second rank \\
\hline 1 & 32 & 10 \\
\hline 2 & 28 & 20 \\
\hline 3 & 13 & 36 \\
\hline 4 & 20 & 27 \\
\hline 5 & 27 & 19 \\
\hline 6 & 0 & 8 \\
\hline 7 & 0 & 30 \\
\hline
\end{tabular}

TABle 1. Dataset of [14].

At the first stage, to find the maximum discriminating factor the proposed Model (7) is solved. By solving this model, the value $\epsilon=0.0020639835$ as the maximum discriminating factor is obtained. At the next stage, we take into account the proposed two scenarios. In this stage, the models (3.2.3) and (3.2.3) are solved. The obtained results of the proposed models alongside the candidates' ranks are summarized in Table 2. Results of the

\begin{tabular}{ccc|cc}
\hline \hline \multirow{2}{*}{ Candidates } & \multicolumn{2}{c|}{ Scenario 1 } & \multicolumn{2}{c}{ Scenario 2 } \\
\cline { 2 - 5 } & Score & Rank & Score & Rank \\
\hline 1 & 1.0000000000 & 1 & 1.0000000000 & 1 \\
2 & 0.9969040246 & 2 & 0.9969040305 & 2 \\
3 & 0.7523219809 & 3 & 0.7523219858 & 3 \\
4 & 0.8498452009 & 4 & 0.8498452061 & 4 \\
5 & 0.9582043342 & 5 & 0.9582043399 & 5 \\
6 & 0.9226006192 & 6 & 0.9226006245 & 6 \\
7 & 0.3250773989 & 7 & 0.3250774013 & 7 \\
\hline \hline
\end{tabular}

TABLE 2. Results of the proposed method.

two scenarios are very close to each other and also they proposed similar rankings. Additionally, the results show that, our proposed models can fully rank candidates, and candidate 1 and 7 are the best and worst candidates, respectively. In order to show the capabilities of the proposed models, we compare our proposed model with other models proposed by [2], [14], [44], Izadikhah and Farzipoor Saen[1], Ebrahimnejad et al. [25] and Izadikhah and Saen [40]. The ranking results of these methods are shown in Table 3. As is seen, Cook and Kress [2] method and Ebrahimnejad et al. [25] method fail to provide a complete ranking. Jahanshahloo et al. [44] method provide an unreasonable result for the first candidate. 


\begin{tabular}{|c|c|c|c|c|c|c|c|c|c|}
\hline \multirow{2}{*}{\multicolumn{4}{|c|}{ Candidate Cook [2] Obata [14] Jahanshahloo [44] }} & \multicolumn{2}{|c|}{ Ref. [1] } & \multicolumn{2}{|c|}{ Ref. [25] } & \multicolumn{2}{|c|}{ Ref. [40] } \\
\hline & & & & Score & Rank & Score & Rank & Score & Rank \\
\hline 1 & 1 & 1 & 5 & 1 & 1 & 1 & 1 & 1 & 1 \\
\hline 2 & 1 & 2 & 1 & 0.87421 & 3 & 1 & 1 & 0.99690 & 2 \\
\hline 3 & 1 & 3 & 2 & 0.41074 & 6 & 0.815789 & 6 & 0.75232 & 6 \\
\hline 4 & 4 & 4 & 3 & 0.62802 & 5 & 0.881579 & 5 & 0.84985 & 5 \\
\hline 5 & 5 & 5 & 4 & 0.84567 & 4 & 0.961111 & 3 & 0.95820 & 3 \\
\hline 6 & 6 & 6 & 6 & 0.93647 & 2 & 0.9375 & 4 & 0.92260 & 4 \\
\hline 7 & 7 & 7 & 7 & 0 & 7 & 0.394737 & $7 \quad 7$ & 0.32508 & 7 \\
\hline
\end{tabular}

TABLE 3. Results of different models.

Using the obtained discriminating factor $(\varepsilon=0.0020639835)$ and given Table 2, unique rankings are obtained which indicate high discrimination powers of our proposed models.

\section{Conclusion}

The key role in social choice and voting systems is played by aggregation of preference orders. The methodologies for solving the voting systems are usually based on determination of an aggregated value for each alternative. In this issue, how to determine the weights associated with rank positions is an important subject since it will determine the final solution. Many researches have been done to develop some procedures in which the weights associated with the votes become variables in the model. Data envelopment analysis represents one class of such models and allows to each DMU to measure its efficiency with the weights that are only most favorable for itself. In the other words, for reaching the maximum efficiency of each DMU, the most favorable weighting schemes are chosen. Thus, the efficiencies of different DMUs obtained by different sets of weights may be unable to be compared and ranked on the same basis. Another problem is that there are always more than one DMU to be evaluated as efficient because of the flexibility in the selection of weights, which would cause the problem that all DMUs cannot be fully discriminated. In order to solve these two problems this paper proposed a new variant of Cook and Kress model with some interesting properties. The proposed model was further extended to the multi-objective programming model to for generating common set of weights. Then, a new model for estimating the maximum discriminating factor among candidates was developed. In order to solve the obtained MOLP model, two scenarios with two linear one-objective models were presented. Solving linear problems was another advantage of the proposed approach against general approaches in the literature which are based on solving nonlinear problems. Compared to the original DEA-based voting model, this approach discriminates in a better way among candidate's in order to yield

the less efficient ones. As in the conventional model, it does not require 
the formulation of $n$ models. In fact, the efficiencies of all candidates can be calculated by solving a single model, enabling one to evaluate the relative efficiency of every candidate on a common weight basis. Considering that the place of each candidate is of great importance from an economic and managerial point of view, various organizations use voting systems and their main objective is to rank candidates. The ranking approach in this paper can be applied in various real-world settings, especially in the business and managerial section. A numerical results not only indicated that the proposed models provide complete ranking among candidates but also validated the correctness of the provided theorems.

\section{REFERENCES}

[1] M. Izadikhah, and R. Farzipoor Saen, A new preference voting method for sustainable location planning using geographic information system and data envelopment analysis, Journal of Cleaner Production, 137: pp. 1347-1367, 2016

[2] W.D. Cook, and M. Kress, A Data Envelopment Model for Aggregating Preference Rankings, Management Science, 36(11): pp. 1302-1310, 1990.

[3] A. Charnes, W.W. Cooper, and E. Rhodes, Measuring the efficiency of decision making units, European Journal of Operational Research, 2(6): pp. 429-444, 1978.

[4] H. Dibachi, M.H. Behzadi, and M. Izadikhah, Stochastic multiplicative DEA model for measuring the efficiency and ranking of DMUs under VRS technology, Indian Journal of Science and Technology, 7(11): pp. 1765-1773, 2014.

[5] M. Izadikhah, R. Farzipoor Saen, and K. Ahmadi, How to assess sustainability of suppliers in volume discount context A new data envelopment analysis approach, Transportation Research Part D: Transport and Environment, 51: p. 102-121, 2017.

[6] A. Kutlar, A. Kabasakal, and M.S. Ekici, Efficiency of commercial banks in Turkey and their comparison: application of DEA with Tobit analysis, Int. J. of Mathematics in Operational Research, 10(1): pp. 84-103, 2017.

[7] R.H. Green, J.R. Doyle, and W.D. Cook, Efficiency of commercial banks in Turkey and their comparison: application of DEA with Tobit analysis, European Journal of Operational Res, 90(3): p. 461-472, 1996.

[8] H. Noguchi, M. Ogawa, and H. Ishii, The appropriate total ranking method using DEA for multiple categorized purposes, Journal of Computational and Applied Mathematics, 146(1): pp. 155-166, 2002.

[9] P.S. Dharmapala, Bias-correction in DEA efficiency scores using simulated beta samples: an alternative view of bootstrapping in DEA, Int. J. of Mathematics in Operational Research, 12(4): pp. 438-456, 2018.

[10] H. Dibachi, M.H. Behzadi, and M. Izadikhah. Indian Journal of Science and Technology, 8(8): pp. 549--555, 2015.

[11] S.M. Mirdehghan, and M. Heydari, Characterisations of the production possibility set in data envelopment analysis: an MOLP approach, Int. J. of Mathematics in Operational Research, 12(1): pp. 31-44, 2018.

[12] P. Andersen, and N.C. Petersen, A Procedure for Ranking Efficient Units in Data Envelopment Analysis, Management Science, 39(10): pp. 1261-1264, 1993.

[13] A. Hashimoto, A ranked voting system using a DEA/AR exclusion model: A note, European Journal of Operational Research, 97(3): pp. 600-604, 1997.

[14] T. Obata, and H. Ishii, A method for discriminating efficient candidates with ranked voting data, European Journal of Operational Research, 151(1): pp. 233-237, 2003.

[15] A.A. Foroughi, and M. Tamiz, An effective total ranking model for a ranked voting system, Omega, 33(6): pp. 491-496, 2005. 
[16] Y.M. Wang, K.S. Chin, and J.B. Yang, Three new models for preference voting and aggregation, Journal of the Operational Research Society, 58(10): pp. 1389-1393, 2007.

[17] Z. Angiz, M.Z., et al., Selecting the most preferable alternatives in group decision making problem using DEA, Expert Systems with Applications, 36: pp. 9599--9602, 2009.

[18] Y.M. Wang, Y. Luo, and Y.X. Lan, ACommon weights for fully ranking decision making units by regression analysis, Expert Syst. Appl., 38(8): pp. 9122-9128, 2011.

[19] F.-H.F. Liu, and H. Hsuan Peng, Ranking of units on the DEA frontier with common weights, Computers \& Operations Research, 35(5): pp. 1624-1637, 2008.

[20] J.A. Ganley, and J.S. Cubbin, Public sector efficiency measurement: applications of data envelopment analysis, Amsterdam: North-Holland, 1992.

[21] C. Kao, and H.T. Hung, Data envelopment analysis with common weights: the compromise solution approach, JJ. Oper Res Soc, 56(10): pp. 1196-1203, 2005.

[22] M. Izadikhah, , A. Saeidifar, and R. Roostaee, Extending TOPSIS in fuzzy environment by using the nearest weighted interval approximation of fuzzy numbers, Journal of Intelligent \& Fuzzy Systems, 27: pp. 2725--2736, 2004.

[23] A. Salehi, and M. Izadikhah, A novel method to extend $S A W$ for decision-making problems with interval data, Decision Science Letter, 3(2014): pp. 225--236, 2014.

[24] Wang, Y.-M. and Y. Luo, DEA efficiency assessment using ideal and anti-ideal decision making units, Applied Mathematics and Computation, 173(2): pp. 90-2-915, 2006.

[25] A. Ebrahimnejad, M. Tavana and F.J. Santos-Arteaga, An integrated data envelopment analysis and simulation method for group consensus ranking, Mathematics and Computers in Simulation, 119: pp. 1-17, 2016.

[26] A. Oukil,and G.R. Amin, Maximum appreciative cross-efficiency in DEA: A new ranking method, Computers \& Industrial Engineering, 81: pp. 14-21, 2015.

[27] Y. Roll, , W. Cook, and B. Golany, Controlling factor weights in data envelopment analysis, IIE Transactions, 24: pp. 1-9, 1991.

[28] Cook, W.D. and M. Kress A multiple criteria decision model with ordinal preference data, European Journal of Operational Research, 137: 54: pp. 191--198, 1991.

[29] Sinuany-Stern, Z. and L. Friedman, DEA and the discriminant analysis of ratios for ranking units, Journal of Cleaner Production, 111: pp. 470--878, 1998.

[30] Sun, J., J. Wu, and D. Guo, Performance ranking of units considering ideal and anti-ideal DMU with common weights, Applied Mathematical Modelling, 37(9): pp. 6301-631, 2013.

[31] S. Saati, and N. Nayebi, An algorithm for determining common weights by concept of membership function, Journal of Linear and Topological Algebra, 4(3): pp. 165-172, 2015.

[32] G.R. Jahanshahloo, et al., A note on some of DEA models and finding efficiency and complete ranking using common set of weights, Applied Mathematics and Computation, 166(2): pp. 265-281, 2005.

[33] W.D. Cook, and J. Zhu, Within-group common weights in DEA: An analysis of power plant efficiency, European Journal of Operational Research, 137: 178(1): pp. 207216, 2007.

[34] J.L. Ruiz, and I. Sirvent, Common benchmarking and ranking of units with DEA, Omega, 137: 65: pp. 1-9, 2016.

[35] M. Carrillo, and J.M. Jorge, A multiobjective DEA approach to ranking alternatives, Expert Systems with Applications, 50: pp. 130-139, 2016.

[36] M. Salahi, N. Torabi, and A. Amiri, An optimistic robust optimization approach to common set of weights in DEA, Measurement, 93: pp. 67-73, 2016.

[37] Tompson, F.D., et al., Tompson, et al., Comparative Site Evaluations for Locating a High Energy Lab in Texas, Intetfaces, pp. 1380-1395, 1986. 
[38] T. Sueyoshi, and M. Goto, A combined use of DEA (Data Envelopment Analysis) with Strong Complementary Slackness Condition and DEA-DA (Discriminant Analysis), Applied Mathematics Letters, 24(7): pp. 1051-1056, 2011.

[39] T. Sueyoshi, and M. Goto, Efficiency-based rank assessment for electric power industry: A combined use of Data Envelopment Analysis (DEA) and DEA-Discriminant Analysis (DA), Energy Economics, 34(3): pp. 634-644, 2012.

[40] Izadikhah, M. and R. Farzipoor Saen, Solving voting system by data envelopment analysis for assessing sustainability of suppliers, Group Decision and Negotiation, 28(3): pp. 641--669, 2019.

[41] M. Toloo, The role of non-Archimedean epsilon in finding the most efficient unit: With an application of professional tennis players, Applied Mathematical Modelling, 38(21-22): pp. 5334-5346, 2014.

[42] M. Toloo, An epsilon-free approach for finding the most efficient unit in DEA, Applied Mathematical Modelling, 38(13): pp. 3182-3192, 2014.

[43] M. Toloo, and A. Kresta, Finding the best asset financing alternative: A DEA-WEO approach, Measurement, 55: pp. 288-294, 2014.

[44] G.R. Jahanshahloo et al., A Method for Discriminating Efficient Candidates with Ranked Voting Data by Common Weights, Mathematical and Computational Applications, 17(1): pp. 1-8, 2012.

(Mohammad Izadikhah) Department Of Mathematics, College Of Science, AraK-Branch, Islamic Azad University, Arak, Iran

Email address: m-izadikhah@iau-arak.ac.ir

(Erdal Karapinar) Department Of Medical Research, China Medical UniverSity, TAICHUnG, TAIWAN.

Email address: erdalkarapinar@yahoo.com 